\title{
Semantic Caseframe Parsing and Syntactic Generality
}

\author{
Philip J. Hayes. Peggy M. Andersen, and Scolt Safier \\ Carnegie Group Incorporated \\ Commerce Court at Station Square \\ Pittsburgh. PA 15219 USA
}

\begin{abstract}
We have implemented a restricted Jomain parser called Plume. $M$ Building on previous work at Carnegie-Melion University e.g. [4, 5. 8]. Plumes approacn to parsing is based on semantic caseframe instantialion This has the advantages of efficiency on grarmatical inout. and robustness in the face of ungrammatical input While Plume is well adapted to simple declarative and imperative utterances. it handles passives relative clauses and interrogatives in an ad hoc manner leading to patchy syntactic coverage This paper outlines Plume as it currently exists and describes our detalled design for extending Plume to handle passives relative clauses. and interrogatives in a general manner
\end{abstract}

\section{The Plume Parser}

Recent work at Carnegie-Mellon University e.g. 14 . 5) has shown semantic caseframe instantiation to be a highly robust and efficient method of parsing restricted domain input. In this approach to parsing. a casetrame grammar contains the domaın-specific semantic information. and the parsing program contains general syntactic knowledge. Input is mapped onto the grammar using this built-in syntactic knowledge we have chosen this approach for Plume ${ }^{-M}$, a commercial restricted domain parser.' because of its advantages in efficiency and robustness

Let us take a simple example from a natural language interface, called NLVMS. that we are developing under a

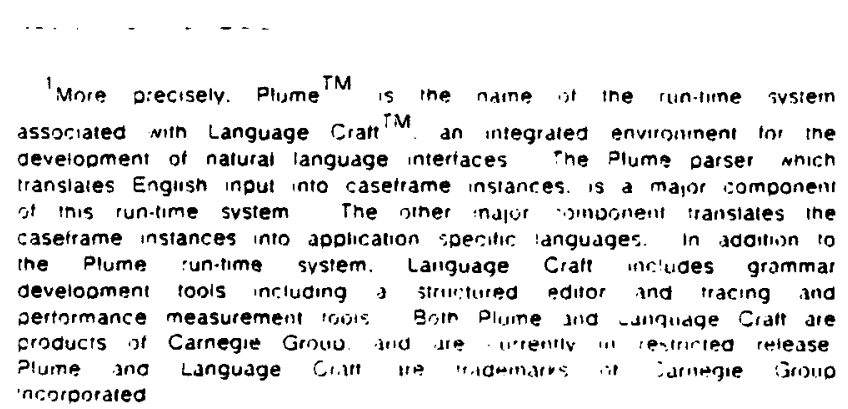

contract with Digital Equipment Corporation NLVMS is an interface to Digital's VMS ${ }^{\nexists}$ operating system for 'VAX ${ }^{\nexists}$ computers. $^{2}$ The Plume grammar for this intertace contains the following semantic casetrame ${ }^{2}$ corresponding 10 the copy command of VMS:

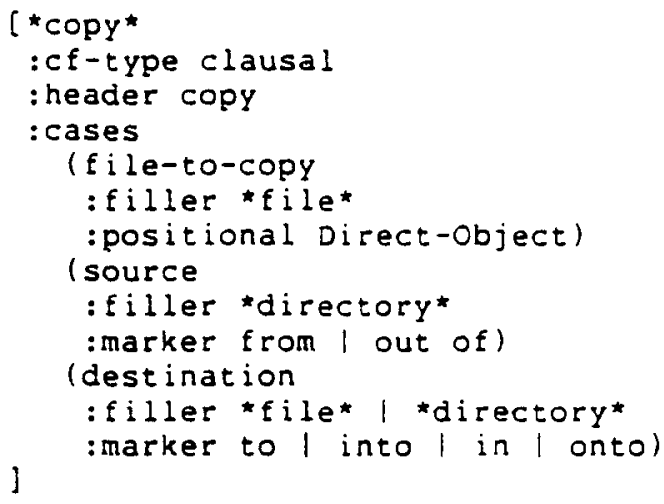

This defines a caseframe called ${ }^{\circ} \mathrm{copy}{ }^{\prime}$ with inree cases file-10-copy, source, and destination the file-10-copy case is filled by an object of type 'file' and appears in the input as a direct object. Source is filled by a "directory" and should appear in the input as a prepositional phrase preceded or marked by the prepositions "from" or "out of" Destination is filled by a "file* or "directory" and is marked by "10". "into". or "onto" Finally the copy command itself is recognized by the header word indicated above (by header) as "copy".

Usıng this caseframe. Plume can parse inputs like:

Cooy too oar out of $[x]$ into $\mid y \Gamma^{2}$

From $[x]$ 10 $[y]$ copy too bar

too oar copy from $[x \mid 10[y]$

\footnotetext{
2VMS and VAX are irademarks of Digital Equipment Corporation

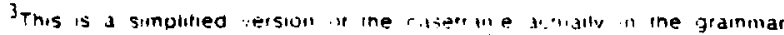


In essence. Plume's parsing algorithm is to find a caseframe header. in this case "copy". and use the associated caseframe. "copy'. 10 guide the rest of the parse. Once the caseframe nas been identified. Plume looks for case markers. and then parses the associated case filler directly following the marker Plume also tries to parse positionally specitied cases. like direct object. in the usual position in the sentence - immediately following the header for direct object. Any input not accounted for at the end of this procedure is matched against any unfilled cases. so that cases that are supposed to be marked can be recognized without their markers and positionally indicated cases can be recognized out of their usual positions. This flexible. interpretive style of matching caseframes against the input allows Plume to deal with the kind of variation in word order illustrated in the examples above.

The above examples implied there was some method to recognize files and directories. They showed only atomic file and directory descriptions. but Plume can also deal with more complex object descriptions. In fact. in Plume grammars. objects as well as actions can be described by caseframes. For instance. here is the caseframe ${ }^{5}$ used 10 define a file for NLVMS.

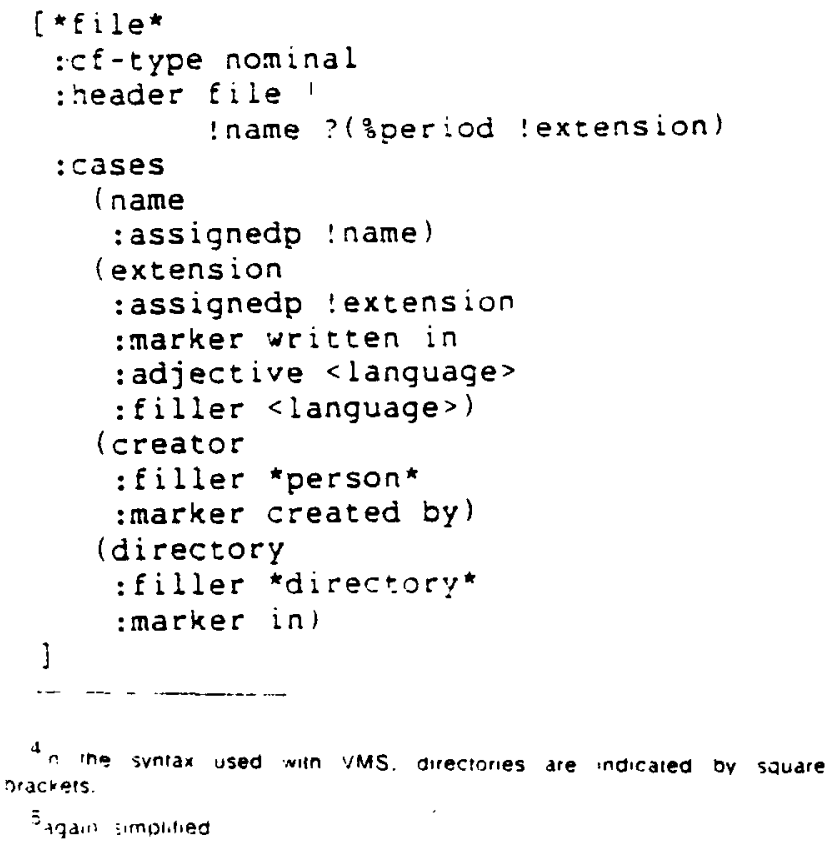

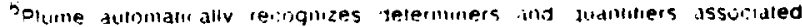
-.in minirial asetrames
This caseframe allows Plume to recognize file descriptions like: 6

100

foo.bar

The file created oy Jonn

The tortran file in $|x|$ created by John

The caseframe notation and parsing algorithm used here are very similar to those described above for clause level input. The significant differences are additions related to the :adjective and assignedp attributes of some of the cases above. While Plume normally only looks for fillers after the header in nominal caseframes an adjective attribute of a slot tells Plume that the stot filler may appear before the header.

An assignedp attribute allows cases to be filled through recognition of a header. This is generally useful for proper names. such as foo and foobar. In the example above. the second alternative header contains iwo variables name and extension. Inat can each match any single vord. The question mark indicates optionality. so inat the header can oe either a single word or a word followed bv a period and another word. The first word is assigned to the variable 'name. and the second if 11 is inerel to the rariable 'extension if 'name or 'exiension are matched while recognizing a file header. their values are placed in the name and extension cases of tile.

With the above modifications Plume can parse nominat caseframes using the same algorithm that it uses for clausal caseframes that account for complete sentences. However there are some interacions between the two levels of parsing. In particular. Inere can be ambiguity about where 10 attach marked cases. For instance. in:

Copy the tortran five in $[x]$ to $|y|$

"In $|x| "$ could either fill the direciorv case of the flle described as "the fortran itle" or could fill the destination case of the whole copy command. The second interpretation does not woik at the global level because the only place to put "to $|y|$ " is in that same destination case However. at the time the file description is parsed. Inis information is not avallable. and so both possible attachments must be considered in general. if Plume is able to fill a case of a nominal caseframe from a 
prepositional phrase. it also splits off an alternative parse in which that attachment is not made. When all input has been parsed. Plume retains only those parses that succeed at the global level. i.e. consume all of the input. Others are discarded.

The current implementation of Plume is based on the nominal and clausal level caseframe instantiation algorithms described above. Using these algorithms and a resiricted domain grammar of caseframes like the ones snown above. Plume can parse a wice variety of imperative and Jeclarative sentences relevant 10 that domain. However. there remain significant gaps in its coverage. Interrogatives are not handled at all " passives are covered only if they are explicilly specified in the grammar and relative clauses can only be handled by pretending iney are a form of prepositional phrase

The regular and prediciable relationship between simple statements. questions and relative clauses and between active and passive seniences is vell known A parser which purports 10 interpret a domain specific language specificalion using a built-in knowledge of syntax snould account for this regularity in a general way the current implementation of Plume has no mechanism for doing inis. Eacn individual possibility for questions. relative clauses and passives must be explicitly specified in the grammar for instance. 10 nandle reduced relative clauses as in "the fite created by jim" "created by" is listed as a case marker lcompound prepositions in the creator siot of file. marking a description of the creator To handle full relatives the case marker must be specified as something like "? (which <be $>$ ) created by". While this allows Plume 10 recognize "the file which was created by Jim". "the file created by Jim". or even "the file created by Jim on Mondav" "breaks down on something like "the file created on Monday by Jim. because the case marker "created by" is no longer a unit. Moreover using the current techniques. Plumes ability 10

\footnotetext{
The current molementation of Plime cumlaus a lemoldary ineinud of

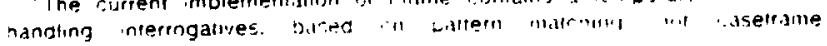
ilitintallun
}

recognize the above inputs is completely unrelated io its ability to recognize inputs like

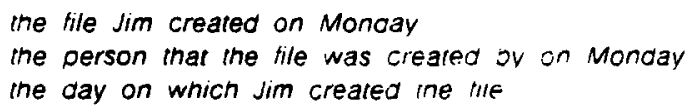

the tile Jim created on Moncay

the person that the file was created ov on Monday

the day on which Jim created ine tile

If an interface could recognize any of these examples it might seem unreasonable to a user that it could not recognize all of the others. Moreover given any of the above examples. a user might reasonably expeci recognition of related sentence level inouts like

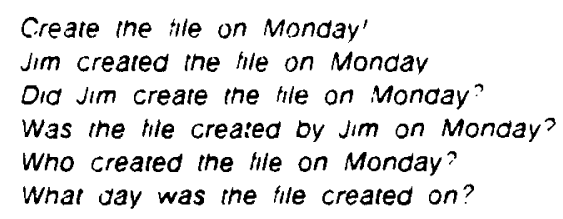

The current implementation of Plume has no means of guaranteeing such regularity of coverage. Of course. this problem of patchy syntactic coverage is not new for restricted domain parsers. The lack of syntactic generality of the original semantic grammar [3] for the Sophie system [2] led to the concept of cascaded ATNS [10] and the RUS parser [1]. A progression with similar goals occurred trom the LIFER system [9] to TEAM [6] and KLAUS [7].

The basıc oostacle to achieving syntactic generality in these network-based approaches was ine way syntacilc and semantic information was mixed logether in the grammar networks. The solutions. theretore. rested on separating the syntacic and semantıc information. plume already incorporates just the separation of syntax and semantics necessary for syntactic generality general syntacilc knowledge resides in the parser. while semantic information resides in the grammar This suggests that svntacilc generality in a system like plume can be achieved by improving the parsers casetrame instaniration aigorithms vincut uny major changes to Jrammar content in terms of the aoove examples involving sreare it suggests ve can use a single 'create' casetrarne to nandte all the examples We simply need 10 provide sultable extensions to the existing caseframe nstantiation algorithms in ine next section we present a delailed design for such extensions

\section{Providing Plume with Syntactic Generality}

As described above. Plume can currently use clausal. 
Note that :marker is the same as theader of create. Similar relative cases are generated in the person. caseframe for the creator case. and in the date caseframe for the creation-date case. differing only in relative-case-name

Relatıve cases are used similarly to the ordinary marked cases of nominal caseframes. In essence. If the parser is parsing a nominal caseframe and finds the marker of one of its relative cases. Inen it tries to instantiate the relativect. It Derforms this instantiation in the same way as if the relative.cf were a rop-level clausal caseframe and the word that matched the header were its main verb. An important difference is that it never tries to fill the case whose name is given by relative-case-name. That case is flled by the nominal caseframe which contains the relative case for instance. suppose the parser is trying to process

The tile Jim created on Monday

Fnd suppose that it has already located "file' and used that to fetermine it is instantiating a 'file. nominat caseframe It is able to match iagainst "created", the marker of the ielative caseframe of 'hle' shown above. It then tries to instantiate the relatwe.cl create using its standard techniques except inat it does not try to fill createe. the case of create specified as the ielalive-casename. This instantiation succeeds with "Jim" going into creator. and "on Monday" being used 10 fill crealion-date The parser then uses la pointer 10 l the nominal caseframe currently being instantiated. 'file ${ }^{\cdot}$ to fill createe. the relative-case-name case of 'create' and the newly created instance of 'create' is attacned to this instance of 'file' as a modifier

More completely. Plume's algorithm for relative clauses is:

1. When processing a nominal caseirame. Plume scans for the :markers of the relative cases of the nominal caseframe at the same lime as it scans for the regular case markers of that nominal casetrame

2. If It finds a marker of a relative case. It iries 10 instąntiate the relalive.cf just as though if were the rop-level clausal casetrarne and the header were its main rero. except inat: a. It never looks any further left in the inout than the neader of the nomiral caseframe or if it has already parsed any orner post-rominal cases of the nominal caseframe no further left than the right hand end of them

b. it consumes. but otherwise ignores any relative pronouns iwho whim whirn inall that immediately precede the segment used 10 instantiate the relative.cf Tnis neans that ali words. Including "Ihar" will be accounted or in "the file inat Jim createc in Monday"

c. it does not try to fill the case specified by the relative-case-name in the relative-ct: instead inis case is filled by la pointer tol the opiginal nominal caseframe instance:

d. If the relative-case-name specifies a marked case rather than a positional one in the relative-cf then its case marker can be consumed. but otherwise ignored. during instantiation of the relative.ct This allows us to deal with "on" in "ine jare Jim creared ine the on" or "the cate un which Jim created the tile.

3. Passive relative clauses le g. "the lile that was created on Monday"' can generally be nandled using the same mechanisms used for passives at the main clause level However. in relative clauses. passives may sometimes be reduced by omiting the usual auxiliary verb 10 be land the relative pronounl as in

the file created on Monday

To account for such reduced relative clauses. the verb cluster processor will produce appropriate additional readings of the verb clusters in relative clauses for which the relative pronoun is missing This may lead to multiole oarses. including one for the above example similar to the correct one for:

the file Jonn created on Monday

These ambiguities will be laken care of by Plumes standard ambiguity reduction methods

\section{3 interrogatives}

In addition 10 handling passives 3nd elative slauses. ise also wish the information in the create sasetrame 10 hangle interrogatives involving "create sucn as

Cid Jim create the file on Momitu'

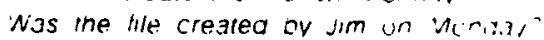

Who created the the on Muncasv.

What day was the the created is

The primary difficulty for Plume aith interrogatives is that. as these examples show. The number of variations in standard constituent order is much greater than for imperatives ano 
declaratives. Interrogatives come in a wide variety of forms. depending on whether the question is yes/no or wh: on which auxiliary verb is used: on whether the voice is active or passive: and for wh questions. on which case is querled. On the other hand. apart from varlations in the order and placement of marked cases. there is only one standard constituent order for imperatives and only two for declaratives (corresponding 10 active and passive voice). We have exploited this low variability by building knowledge of the imperative and declarative order into Plume's parsing algorithm. However this is impractical for the larger number of variations associated with interrogatives. Accordingly, we have designed a more data-driven approach.

This approach involves two passes through the input: the first categorizes the input into one on several primary input categories including yes-no questions. several kinds of whquestions. statements, or imperatives. The second pass performs a detarled parse of the input based on the classification made in the first pass. The rules used contain basic syntactic information about Engish. and will remain constant for any of Plume's restricted domain grammars of semantic casetrames for English

The first level of processing involves an ordered sel of wo-level patterns. Each 10D-leyel oattern corresponds to one of the primary input categories mentioned aoove. This classificatory matching does not dttempt to match every word in the input sentence. but only to do the minimum necessary to make the classification. Most of the relevant information is found at the beginning of the inputs. in particular. the lop-level patterns make use of ine fronted auxiliary verb and wh-words in questions.

As well as classifying the input. this top-level match is also used to determine the identity of the caseframe to be instantiated. This is important is do at this stage because the detailed recognition in the second phase is heavily dependent on the identity of inis top-level caseframe the special symbol. svero. that appears exactiy once in all ioplevel patterns. matches a header of any clausal caseframe We call the casetrame whose header is matched by Svero the primary casetrame for that input.
The second more detailed parsing phase is organized relative to the primary caseframe. Associated with each toplevel pattern, there is a corresponding parse template. A parse template specifies which parts of the primary caseframe will be found in unusual positions and which parts the default parsing process (the one for declaratives and imperatives) can be used for.

A simplified example of a top-level pattern for a yes-no question is: ${ }^{11}$

<aux> C (\$vero!! <aux>H) (\&s sverb) Srest

This top-level pattern will match inputs like. the following:

Did Jim create too?

Was too created oy Jim?

The first element of the above top-tevel pattern is an auxiliary verb. represented by the non-terminal <aux> This auxiliary is remembered and used by the verb cluster processor (as though it were ine first auxiliary in the cluster) to determine tense and voice. According to the next part of the pattern. some word that is not a vero or an auxiliary must appear after the fronted auxiliary and before the main verb $($ - is the negation operator. and !! marks a disjunction). Next. The scanning operator \&s tells the matcher to scan until it finds svero. which matches the header of any clausal casetrame Finally. \$rest matches the remaining input.

If the top-level pattern successfuily matches. Flume uses the associated parse template to direct its more detalled processing of the input. The goal of this second pass through the inpul is to instantiate the caseframe corresponding to the header matched by Sverb in the 100. level pattern. The concept of a kernel-casetrame is important to this stage of processing. A kernel-caseframe corresponds 10 that part of an input that can be processed according to the algorithm already built into plume for declarative and imperative sentences.

\footnotetext{
' 'In this Dattern. only mouts where the tronied auxillary is ine irs whro in the sentence dre allowed the more monolex pallem ind is

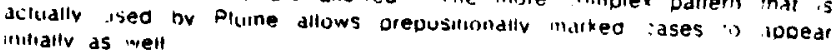


The parse template associated with the above top-level pattern for yes/no questions is:

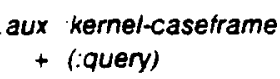

This template tells the parser that the input consists of the auxiliary verb matched in the first pass followed by a :kernel-caseframe. For example. in

Dio Jim create too?

the auxtliary verb. "did". appears first followed by a kernelcaseframe. "Jim create foo" Note now the kernel. caseframe looks exactly like a declarative sentence, and so can be parsed according to the usual declarative/imperative parsing algorithm

In addition to specification of where to find components of the primary caseframe. a parse template includes annotations (indicated by a plus sign) in the above template for yesino questions. there is just one annotation query. Some annotations. like this one indicate what type of input has been found. while others direct the processing of the parse template. Annotations of the first type record which case is being queried in wh questions. that is. which case is associated with the wh word. Wh questions thus include one of the following annotations sublect-query. ooject-query, and marked-case-query Marked case queries correspond to examples like

On what day did Jim create too? What day did Jim create too on?

in which a case marked by a preposition is being asked about. As illustrated here the case-marker in such queries can elther precede the wh word or appear somewhere after the verb. To deal with this. the parse template for marked case queries has the annotation moatino-case-marker. This annotation is of the second type inat is 11 affects the way Plume processes the associated parse template.

Some top-level patterns result in two possibilities for parse templates. For examole. the following top-level pattern

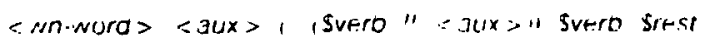

could match an object query or a marked case query. including the following:

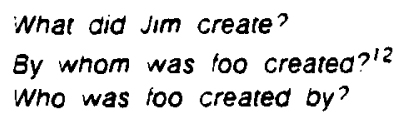

These inputs cannot be satisfactorily discriminated by a loplevel pattern. so the above top-level pattern has two different parse templates associated with it:

wh-object aux kernel-casetrame + (obiect-query)

wh-marked-case-filler aux kernel-casetrame + (marked-case-query floaring-case-marker).

When the above top-level pattern matches. Plume tries to parse the input using both of these parse templates. In general. only one will succeed in accounting for all the inpul. so the ambıguity will be eliminated by the methods aiready built into Plume

The method of parsing interrogatives presented above allows Plume to nandle a wide variery of interrogatives in a very general way using domain specific semantic caseframes. The writer of the caseframes does not have 10 worry about whether they will be used for imperative, declarative. or interrogative sentences. (or in relative clauses). He is free to concentrate on the domain-specific grammar. In addition. the concept of the kernel-caseframe allows Plume to use the same efficient caseframe-oased parsing algorithm that it used for dectarative and imperative sentences 10 parse major subparts of questions.

\section{Conclusion}

Previous work (e.g. [4. 5. 31) and experience with our current implementation of Plume. Carnegie Grouos semantic caseframe parser. has snown semantıc caseframe instantiation to be an efficient and highly robust method of parsing restricted domain noul However like other methods of parsing heavily Jependent on restricted domain semantics. these initial attempts at parsers based on semantic caseframe instantiation suffer from patchy syntactic coverage. 
After first describing the current implementation of Plume. this paper presented a detalled design for endowing Plume with much broader syntactic coverage including passives. interrogatives, and relative clauses. Relative clauses are accommodated through some grammar preprocessing and minor change in the processing of nominal caseframes Handling of interrogatives relies on a set of rules for classifying inputs into one of a limited number of types. Each of these types has one or more associated parse templates which guide the subsequent detailed parse of the sentence. As the finat version of this paper is prepared (late April. 1985). the handling of passives and interrogatives has already been implemented in an internal development version of Plume. and relative clauses are expected to follow soon

Though the above methods of incorporating syntactic generality into Plume do not cover all of Engtish syntax. they show that a significant degree of syntactic generality can be provided straightforwardly by a domain specific parser driven from a semantic caseframe grammar

\section{References}

1. Bobrow. R J. The RUS System BBN Report 3878. Bolt. Beranek. and Newman. 1978

2. Brown. J. S. and Burton. A R Multiole Representations of Knowledge for Tutorial Reasoning. In Representation and Undersianding. Bobrow. D. G and Collins. A.. Ed. Academic Press. New York. 1975. pp $311-349$.

3. Burton, R. R. Semantic Grammar An Engineering Technique for Constructing Natural Language Understanding Systems. BBN Report 3453. Bolt. Beranek. and Newman. Inc.. Cambridge. Mass.. December. 1976.

4. Carbonell, J. G. Boggs. W. M. Mauidin. M. L.. and Anick, P. G. The XCALIBUR Projec: A Natural Language Interface to Expert Systems. Proc. Eighth int. Jt. Conf. on Artificial Intelligence. Karlsrune. August. 1983

5. Carbonell. J. G. and Hayes $P J$. "Recovery Sirategies for Parsing Extragrammatical Language". Computational Linguistics 10 (1984).

6. Grosz. B. J. TEAM: A Transportable Natural Language Interface System. Proc. Conf on Applied Natural Language Processing. Santa Monica. February 1983

7. Haas. N. and Hendrix. G. G. An Approach to Acquiring and Applying Knowledge Proc. Natonal Conference of the American Association for Artificial Intelligence. Stanford University. August. 1980. pp. 235.239

8. Hayes. $P$ J. and Carbonell. J G. Muiti-Strategy Parsing and its Role in Robust Man-Machine Communication Carnegie-Mellon University Computer Science Department. May. 1981

9. Hendrix. G. G. Human Engineering for Applied Natural Language Processing. Proc Fifth Int. Jt. Conf on Arrificia intelligence. MIT. 1977. pD. 183.191

10. Woods. W. A. "Cascaded ATN Grammars" American Journal of Computational Linguistics 6. 1 (August 1980). $1-12$ 